\title{
Descuento temporal y probabilístico de dinero y alcohol de usuarios en tratamiento
}

\section{Delay and probabilistic discounting of money and alcohol of users in treatment}

\author{
Juan Carlos González, Raúl Ávila y Silvia Morales-Chainé \\ Universidad Nacional Autónoma de México, Ciudad de México, México
}

\begin{abstract}
Resumen: Una estrategia para estudiar la conducta impulsiva de las personas es el procedimiento de descuento del valor relativo de las recompensas. Se ha reportado que las personas que abusan del consumo de alcohol descuentan más el valor de una recompensa en función de su demora o de las probabilidades de entrega, en comparación con las personas sin problemas de consumo. En el presente trabajo se probó si las tasas de descuento temporal y probabilístico contribuyen a diagnosticar el perfil del bebedor problema. Se expuso a 25 bebedores con indicadores de abuso, a una tarea de descuento temporal y a una tarea de descuento probabilístico de dinero o de una cantidad de la bebida preferida del usuario. En el descuento temporal se encontró que la tasa de descuento fue ligeramente mayor para el alcohol que para el dinero; las tasas de descuento probabilístico fueron muy similares para ambos tipos de recompensa. Los resultados del presente estudio, además de extender los hallazgos previos acerca de las diferencias en el valor asignado a las recompensas en función de su demora o su probabilidad de entrega, también contribuyen a aclarar la relación entre los diferentes procesos involucrados en el descuento del valor psicológico de una recompensa.
\end{abstract}

Palabras clave: descuento temporal, descuento probabilístico, elección, alcohol, humanos.
Abstract: A strategy to study impulsive behavior is the procedure of discounting the relative value of rewards. It has been reported that alcohol abusers show steeper delay or probabilistic discounting of a reward compared to people without drinking problems. The present study examined if the delay and probabilistic discounting rates contribute to diagnose the profile of the problem drinkers. A sample of 25 alcohol abusers completed delayed and a probabilistic discounting tasks of a certain amount of money or of their favorite drink. It was found that the delay discounting rate was slightly higher for alcohol than for the money; in the case of the probabilistic discounting task, the rates were similar for both rewards. The results of this study besides extending previous findings about the differences in the value assigned to different rewards based on their probability or delay of delivery also help to clarify the relationship between the various processes involved in the psychological value of a reward.

Keywords: delay discounting, probabilistic discounting, choice, alcohol, humans.

El presente trabajo corresponde a la versión corta de la tesis para obtener el grado de Maestro en Psicología que presentó el primer autor; el segundo y tercer autores participaron como director y revisor de la tesis, respectivamente. La elaboración final de este manuscrito fue apoyada parcialmente por el subsidio PAPIIT $\mathrm{N}^{\circ}$ IN 303213, otorgado por la DGAPA de la Universidad Nacional Autónoma de México, UNAM, al segundo autor. 
Contacto: J. C. González, Facultad de Psicología. Avenida Universidad 3004, Copilco Universidad, Primer Piso, Edificio D., Lab. 16, 04510, Ciudad de México, México. Correo electrónico: gonzalez.jc16@gmail.com

Cómo citar: González, J. C., Ávila, R. y Morales-Chainé, S. (2015). Descuento temporal y probabilístico de dinero y alcohol de usuarios en tratamiento. Revista de Psicología, 24(1), 1-14 http://dx.doi.org/10.5354/0719-0581.2015.36869 


\section{Introducción}

En su Reporte mundial sobre el alcohol y la salud, la Organización Mundial de la Salud (2014) plantea que el abuso en el consumo de alcohol se ha convertido en un problema a nivel mundial. Entre las principales consecuencias asociadas con el abuso y la dependencia al alcohol, se han propuesto distintos tipos de cáncer, problemas económicos, sociales, personales y psicológicos (Franck y JayaramLindström, 2013). En México, de acuerdo con la Encuesta nacional de adicciones (Instituto Nacional de Psiquiatría Ramón de la Fuente Muñiz, Instituto Nacional de Salud Pública y Secretaría de Salud, 2012), en los últimos años el consumo de alcohol per cápita aumentó, mientras que la edad de inicio de consumo disminuyó, incrementando la probabilidad de desarrollar un comportamiento adictivo a la sustancia desde temprana edad. De acuerdo con el Manual diagnóstico y estadístico de los trastornos mentales (DSM-IV, por sus siglas en inglés), la conducta adictiva "consiste en un grupo de síntomas cognoscitivos, comportamentales y fisiológicos que indican que el individuo continúa consumiendo la sustancia, a pesar de la aparición de problemas significativos relacionados con ella" (American Psychiatric Association, 2000, p. 182). Conductualmente se presenta un patrón de consumo repetido que lleva a la persona a desarrollar tolerancia, síndrome de abstinencia e ingestión compulsiva de la sustancia. De acuerdo con esta definición se pueden destacar dos comportamientos característicos de los consumidores de alcohol: la impaciencia y la propensión a involucrarse en situaciones de riesgo, ambas relacionadas con la obtención o el consumo de la sustancia.

De acuerdo con Bickel, Odum y Madden (1999), la conducta adictiva puede con- ceptualizarse como un problema de elección entre comportarse de manera autocontrolada, o bien, de manera impulsiva o impaciente. Se ha propuesto que una estrategia para estudiar la conducta impulsiva es el método de descuento temporal, con el cual se averigua la manera en que una recompensa pierde su valor subjetivo en función de su demora de entrega. Para determinar el valor subjetivo de una recompensa se pide a los participantes que elijan entre pares de recompensas que varían en magnitud y en demora de entrega: por ejemplo, entre una recompensa relativamente chica e inmediata y una recompensa grande, pero demorada. En elecciones sucesivas se ajusta la magnitud de la recompensa inmediata, manteniendo fijo el valor de la recompensa demorada hasta que el participante elige indistintamente cualquiera de las dos recompensas. Ese punto se conoce como punto de indiferencia y representa el valor psicológico de la recompensa demorada para esa demora específica (Odum y Rainaud, 2003). Al graficar los puntos de indiferencia para cada demora se obtiene una curva de descuento que muestra qué tan rápido pierde su valor una recompensa en función de su demora de entrega. Se ha propuesto que la ecuación hiperbólica es la función que mejor describe la curva de descuento (Rachlin, Raineri y Cross, 1991).

$$
V=\frac{A}{(1+k D)} \quad \text { (Ecuación 1) }
$$

Aquí $V$ representa el valor psicológico de la recompensa; $A$ representa el valor no descontado de la misma; $D$ es la demora expresada en días; y $k$ es un parámetro que indica la tasa de descuento. La elección de la recompensa pequeña e inmediata produce valores de $k$ altos y es un indicador cuantitativo de la conducta impulsiva. La elección de la recompensa 
grande y demorada resulta en valores bajos del parámetro $k$ y muestran una conducta autocontrolada. Esta distinción entre conducta impulsiva y controlada se verifica dado que después de una serie de elecciones, preferir la recompensa inmediata resulta en una magnitud de la recompensa menor, comparada con la magnitud resultante de elegir la recompensa demorada (Logue, 1988).

En diferentes estudios se ha empleado el procedimiento de descuento temporal y la ecuación hiperbólica para describir la manera en la que usuarios y no usuarios de sustancias adictivas juzgan el valor subjetivo de recompensas hipotéticas, como una forma de comparar si el consumo de sustancias está relacionado con una mayor conducta impulsiva (para una revisión ver Yi, Mitchell y Bickel, 2010). Por ejemplo, Petry (2001) expuso a usuarios problemáticos de alcohol y a personas sin historia de consumo de drogas a una tarea de descuento temporal dividida en dos partes. En la primera, los participantes eligieron entre recompensas monetarias hipotéticas y en la segunda, entre cantidades hipotéticas de bebidas alcohólicas. La autora encontró que todos los participantes descontaron más el valor de bebidas alcohólicas en comparación con las recompensas económicas. Además, los consumidores problemáticos de alcohol descontaron con mayor tasa ambos tipos de recompensa, en comparación con los participantes sin historia de consumo de sustancias. Petry planteó que posiblemente los patrones de conducta característicos en consumidores problema de bebidas alcohólicas, como la constante búsqueda de la bebida para escapar del síndrome de abstinencia, podrían provocar que la tasa de descuento del alcohol fuera mayor.

Por su parte, Odum y Rainaud (2003) reportaron que personas sin historia de problemas de consumo de sustancias adictivas descontaron más rápido el valor del alcohol y de la comida en comparación con el valor del dinero. Las autoras sugirieron que las diferencias en las tasas de descuento se deben a las características de los estímulos -el dinero es un reforzador condicionado no consumible y el alcohol es un reforzador consumible, al igual que la comida-, y no a las características de los participantes (como consumir sustancias adictivas). En tanto, Estle, Green, Myerson y Holt (2007), al igual que Odum y Rainaud, encontraron que el valor psicológico de recompensas consumibles (alcohol, comida o refresco) disminuyó más rápido que el valor del dinero, por lo que propusieron que se descontaban menos los reforzadores condicionados como el dinero, porque a la larga pueden ser cambiados por un bien primario que se necesite en el momento que se entrega la recompensa, satisfaciendo más de una necesidad. Por el contrario, los reforzadores primarios o consumibles solo pueden satisfacer una necesidad específica, por ejemplo, el hambre con comida o la sed con agua.

Paralelamente, se ha propuesto el descuento probabilístico como un método para estudiar la propensión a involucrarse en conductas de riesgo. En este caso se descuenta el valor de una recompensa conforme incrementa la razón de probabilidades de obtenerla $(\theta=(1-p) / p)$. Se ha planteado también que al igual que con el descuento temporal, aplicar una probabilidad de entrega a una recompensa produce cambios en el valor subjetivo de la misma, por lo cual es posible describir el descuento probabilístico con una ecuación hiperbólica (Rachlin et al., 1991):

$$
V=\frac{A}{(1+k \theta)} \quad(\text { Ecuación 2) }
$$


Aquí los valores de $V$ y $A$ representan el valor psicológico y el valor no descontado de la recompensa, respectivamente; $\theta$ representa la razón de probabilidades; y el parámetro $k$ nuevamente indica la tasa de descuento. Valores altos de $k$ están asociados con la elección de recompensas grandes, pero con probabilidades bajas de obtenerlas, es decir, propensión a conductas de riesgo. Valores del parámetro $k$ cercanos a cero indican elecciones de recompensas pequeñas, pero con mayor probabilidad de obtenerlas, por lo que se asocian con la aversión a involucrarse en situaciones de riesgo.

Los participantes del estudio de Estle et al. (2007) también fueron expuestos a un procedimiento de descuento probabilístico del valor de dinero, comida, refresco y bebidas alcohólicas; los autores no encontraron diferencias entre las tasas de descuento probabilístico de las diferentes recompensas, proponiendo que posiblemente involucrar la incertidumbre en la entrega de una recompensa produce que las personas evalúen de igual forma el valor de las mismas, independientemente de su naturaleza.

De acuerdo con las descripciones de los patrones conductuales de las personas con consumos problemáticos de bebidas alcohólicas, dos de sus principales características son la impaciencia por beber y el comportarse de manera riesgosa para conseguir la bebida; ambos tipos de conducta han sido ampliamente estudiados con los métodos de descuento temporal y probabilístico, respectivamente. De este modo, la tasa de descuento temporal de un bebedor problemático debería ser alta, asociado con una conducta impulsiva; mientras que su tasa de descuento probabilístico debería ser baja, valor asociado con la propensión al riesgo (Green y Myerson,
2013). Por lo tanto, en el presente trabajo se investigó si las tasas de descuento temporal y probabilístico de alcohol y dinero contribuyen a determinar el perfil del bebedor problema. A pesar de que se ha probado que usuarios y no usuarios de alcohol descuentan más el valor del alcohol que del dinero en el descuento temporal, hasta donde los autores tienen conocimiento, no se ha probado si los usuarios de alcohol descuentan de manera diferente de los no usuarios una sustancia adictiva $y$ un reforzador condicionado generalizado en un procedimiento de descuento probabilístico. En el presente estudio también se exploró esta posibilidad.

\section{Método}

\section{Participantes}

Participaron 25 personas de entre 17 y 45 años de edad diagnosticadas, por lo menos, como bebedores problemáticos (16 puntos o más en el AUDIT, Organización Mundial de la Salud, 2001) que acudieron a un Centro de Prevención de Adicciones de una universidad pública. De los participantes, 15 fueron hombres y 10 mujeres y todos firmaron una carta de consentimiento informado de participación al inicio del estudio.

\section{Instrumentos}

Se entrevistó a cada participante para determinar su nivel de adicción, su bebida de preferencia y el precio por bebida. A cada participante se le aplicó una prueba de alcoholemia mediante un alcoholímetro, para determinar si asistieron sin consumo a la sesión de evaluación; en caso de presentarse a la sesión bajo los efectos del alcohol o de cualquier otra sustancia adictiva (excepto tabaco), se descartó a dicha persona para el estudio. 
Se utilizó un computador personal (DELL Vostro 470, Windows 7) equipado con un monitor de pantalla plana de 20" (DELL E2014H), en la cual se programó una tarea que consistió en la presentación de una serie de elecciones entre recompensas que variaban en magnitud y demora de entrega (descuento temporal), o bien, en magnitud y en probabilidad de entrega (descuento probabilístico). Como operandos los participantes podían utilizar el teclado (DELL: 331-2250) o el ratón (DELL MS111) del computador. Todos los eventos experimentales se programaron y registraron con el software JAVA. La sesión experimental se condujo de manera individual en un cubículo de $2 \times 3$ metros con iluminación artificial, aislado de ruidos externos. La duración de la sesión, incluyendo la entrevista y la prueba de alcoholemia duró aproximadamente 70 minutos.

\section{Procedimiento}

Después de responder la entrevista inicial y firmar el consentimiento de participación, se pidió a los participantes que contestaran una tarea de descuento temporal y una de descuento probabilístico, ambas en un computador. Se dividió la tarea de descuento en dos partes, las que se describen a continuación.

La primera parte de la tarea consistió en una serie de elecciones entre pares de recompensas económicas, para obtener los puntos de indiferencia de descuento temporal y de descuento probabilístico para cada participante. Para el descuento temporal, los participantes eligieron entre una cantidad de dinero disponible de inmediato y $\$ 1000$ MXP (aproximadamente $\$ 650$ USD o €600) disponibles después de cierta demora. Para el descuento probabilístico los participantes eligieron entre una cantidad de dinero segura o $\$ 1000$ entregados con cierta probabilidad de obtenerla. El valor de la recompensa inmediata o segura podía variar entre $\$ 50$ y $\$ 1000$ en intervalos de $\$ 50$. Se determinaron los puntos de indiferencia para seis demoras $(1,2$, $7,30,180$ y 365 días) y para seis probabilidades de obtener la recompensa $(.95, .90$, $.75, .50, .25$ y .05 , expresados como porcentajes); la tarea se programó para que la presentación de las demoras y las probabilidades fuera aleatoria.

Durante la segunda parte de la tarea, se pidió a los participantes que eligieran entre pares de recompensas consumibles, en este caso, su bebida alcohólica preferida. En la evaluación cada participante indicó su bebida de preferencia y el precio de la misma. Con estos datos se obtuvo el precio por copa estándar (aproximadamente $12 \mathrm{~g}$ del alcohol puro) para presentar a cada participante una serie de elecciones y determinar los puntos de indiferencia para un número de copas estándar de bebida alcohólica equivalente a \$1000 (Odum y Rainaud, 2003). Por ejemplo, si un participante mencionaba que consumía cerveza (una copa estándar) y que cada una le costaba $\$ 25$, la cantidad máxima de cervezas que podía comprar era 40; por lo que 40 era el valor de la recompensa demorada o probable. Los valores de la recompensa inmediata o segura variaron con la cantidad de copas estándar que se pueden comprar con $\$ 50$; en el ejemplo equivalen a dos cervezas.

Al iniciar la sesión experimental a cada participante se le leyeron las siguientes instrucciones:

A continuación tendrá que elegir entre diferentes cantidades de dinero disponible después de diversas demoras o con variadas probabilidades de obtenerlo. Las preguntas que se le presentarán son como las que están en la 
pantalla, por ejemplo, “QQué prefiere? $\$ 500$ hoy o $\$ 1000$ dentro de 180 días", o también “¿Qué prefiere? \$300 con el $100 \%$ de certeza de obtenerlos, o bien, $\$ 1000$ con el $90 \%$ de certeza de obtener esa cantidad". También se le presentarán preguntas que no incluyen recompensas económicas, sino recompensas consumibles, en este caso alcohol. Por ejemplo, “¿Qué prefiere? 20 cervezas hoy o 40 cervezas dentro de 180 días”, o también, “QQué prefiere? 10 cervezas con el $100 \%$ de probabilidad de obtenerlas o 40 cervezas con el $50 \%$ de probabilidades de obtenerlas. Para elegir una de las dos recompensas haga clic con el ratón del computador sobre el cuadro que representa su elección. Le recordamos que en ambos casos las recompensas son hipotéticas, por lo que no se le entregará ninguna de ellas al final de la evaluación, pero le pedimos que haga sus elecciones como si fuera a recibir cada una de las recompensas. Por favor, elija la opción que usted prefiera, no la que usted piense que otro preferiría. No esperamos que elija una recompensa u otra, solo elija lo que usted realmente quiere.

Después de leer las instrucciones se expuso al participante a cinco ensayos de prueba para asegurarse de que había entendido la tarea. Si no había ninguna duda, iniciaba la tarea con el procedimiento que se describe a continuación.

Se utilizó el método reportado por Richards, Zhang, Mitchell y de Witt (1999) en el cual se ajustó la magnitud de la recompensa inmediata o probable conforme transcurrieron los ensayos de elección, hasta obtener los puntos de indiferencia entre la recompensa inmediata y la recompensa demorada para cada demora programada, o bien, entre la recompensa segura y la recompensa probable en el caso del descuento probabilístico.

\section{Resultados}

En la figura 1 se presentan las medianas de los puntos de indiferencia obtenidas a partir de las elecciones de los 25 participantes para el dinero (triángulos) y para el alcohol (círculos) en función de su demora (lado izquierdo) o de las probabilidades en contra de obtener la recompensa (lado derecho). Las líneas punteadas representan el ajuste de los datos con la Ecuación 1 o 2 para el descuento del dinero y las líneas sólidas representan el ajuste con la misma ecuación para el alcohol. Los valores de los puntos de indiferencia están expresados en pesos, dado que la recompensa demorada fue $\$ 1000$ o su equivalente en copas estándar (e.g., cuántas cervezas puede comprar un usuario con $\$ 1000$ ).

En el caso del descuento temporal, los participantes descontaron el valor de ambas recompensas conforme aumentó su demora de entrega, pero el alcohol perdió su valor más rápido en comparación con el dinero. Por ejemplo, \$1000 demorados una semana fueron psicológicamente equivalentes a $\$ 400$, mientras que $\$ 1000$ expresados en bebidas alcohólicas fueron equivalentes a $\$ 250$ aproximadamente. Se encontró que la tasa de descuento para la recompensa económica fue menor $(k=$ 0.06; IC 95\% [0.0062, 0.1132]) en comparación con la tasa de descuento para el alcohol $(k=0.204 ;$ IC 95\% [-0.0893, 0.4977]). En el caso del descuento probabilístico los participantes descontaron ambas recompensas con tasas de descuento similares $(k=2.75$, IC 95\% [0.5713, 4.928] y $k=2.145, I C 95 \%$ [1.055, 3.235] para dinero y para alcohol, respectivamente). 


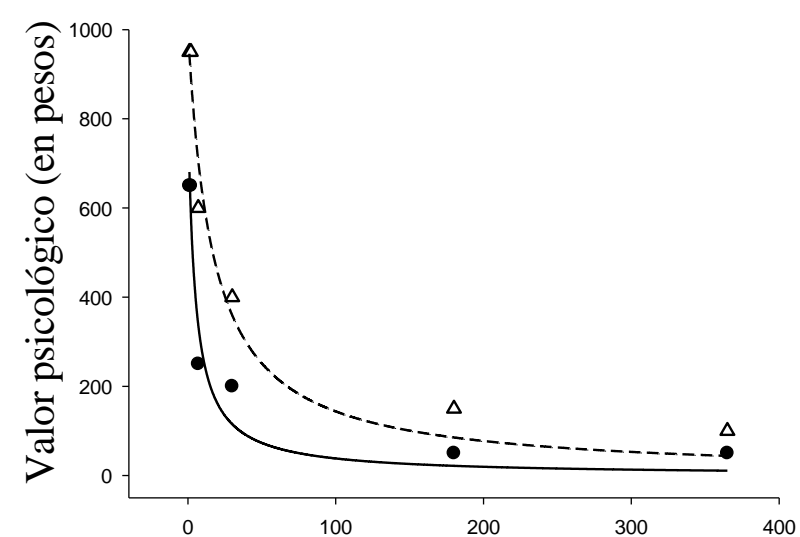

Demora (días)

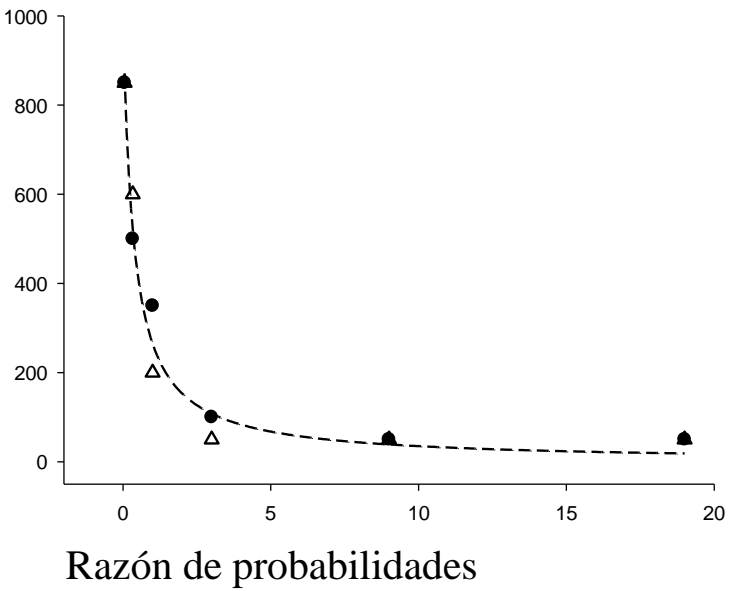

Figura 1. Medianas de los puntos de indiferencia para el descuento temporal (panel izquierdo) y el descuento probabilístico (panel derecho) de dinero (triángulos) y alcohol (círculos). Las líneas representan el ajuste con la ecuación hiperbólica.

Para comparar si los consumidores de alcohol descuentan de manera distinta las recompensas demoradas o las recompensas probabilísticas fue necesario obtener las tasas de descuento de cada participante para ambos tipos de recompensa. Sin embargo, al igual que en otros reportes, se encontró que el modelo hiperbólico no describe adecuadamente los puntos de indiferencia de todos los participantes (Odum y Rainaud, 2003). Una propuesta alternativa para estimar las tasas de descuento es el área bajo la curva -una medida teóricamente neutral-, por lo que no es necesario que los datos se ajusten a un modelo matemático y puede calcularse independientemente de la forma de la curva de indiferencia (Myerson, Green y Warusawitharana, 2001). De acuerdo con dichos autores, las áreas bajo la curva cercanas a cero indican mayor descuento y áreas bajo la curva cercanas a uno implican menor descuento. Al obtener el área bajo la curva de cada participante para ambos tipos de descuento, los autores del presente trabajo notaron que en algunos casos, los participantes no descontaron el valor de las recompensas en función de su demora o de su probabilidad de entrega.

En otros estudios se han reportado estos últimos casos como ejemplos de descuento no sistemático (Johnson y Bickel, 2008), el cual podía deberse a que el participante no entendió la tarea, a que no hizo contacto con la misma o simplemente que la contestó sin atender a ella. Dichos autores propusieron dos reglas con las cuales se puede identificar si un participante no descuenta sistemáticamente el valor de una recompensa. Primero, que cualquier punto de indiferencia, a partir de la segunda demora o probabilidad, sea mayor en comparación con el punto anterior con una magnitud del $20 \%$ de la recompensa demorada. Segundo, que el último punto de indiferencia no sea menor al punto de la demora más corta, con una magnitud de, al menos, el $10 \%$ de la recompensa demorada. La violación de la primera regla indicaría que, para un participante determinado, el valor de una recompensa se incrementa en vez de disminuir conforme incrementa su demora o la razón de probabilidades de entrega. 
La violación de la segunda regla indica que el participante está siendo indiferente a la demora o a la probabilidad, por lo que el valor que asigna a una recompensa es el mismo, sin importar las condiciones de entrega. Para comparar si los participantes descontaron más el dinero que el alcohol o viceversa, fue necesario que mostraran un descuento sistemático en ambos tipos de recompensa; de los 25 participantes, 13 descontaron sistemáticamente ambos tipos de recompensa en función de su demora de entrega. Para el descuento probabilístico se encontró que 11 de los 25 participantes descontaron sistemáticamente el valor de ambas recompensas. Las medias de las áreas bajo la curva calculadas con los participantes que mostraron descuento sistemático en ambas recompensas se presentan en la figura 2.
En la figura, las barras blancas representan las medias del área bajo la curva para el descuento temporal de alcohol y dinero, mientras que las barras negras representan la misma variable para el descuento probabilístico de ambas recompensas. En el descuento temporal se observó que el área bajo la curva para el dinero fue de 0.24 y el área bajo la curva del alcohol de 0.18. Se compararon los datos con una prueba $\mathrm{T}$ para muestras relacionadas, con la cual se encontró que no hay diferencias significativas entre el descuento de las dos recompensas $(t=1.457 ; g l=12 ; p=0.171)$. Para el descuento probabilístico se encontró que las áreas bajo la curva fueron muy similares entre sí (0.15 para el dinero y 0.16 para el alcohol); una prueba $\mathrm{T}$ para muestras relacionadas confirmó la ausencia de dichas diferencias $(t=-0.367$; $g l=10 ; p=0.721)$.

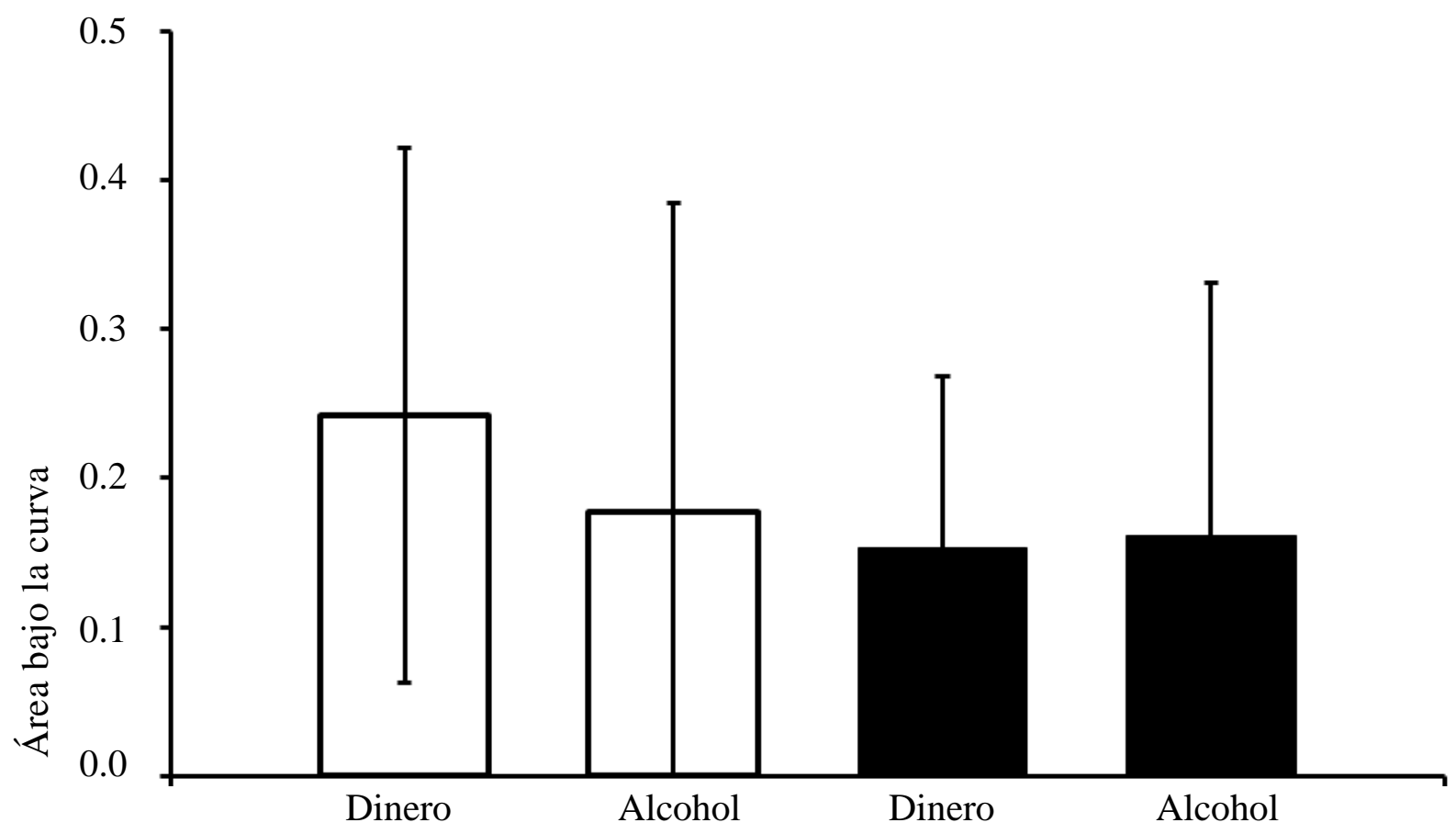

Figura 2. Medias del área bajo la curva para el descuento temporal (barras blancas) y el descuento probabilístico (barras negras) de alcohol y dinero. Se presentan los datos de los participantes que mostraron descuento sistemático en ambas recompensas. 
Como se mencionó anteriormente, se ha propuesto que la interacción entre las tasas de descuento temporal y probabilístico podría servir como un predictor de la conducta adictiva (Green y Myerson, 2013). Así, una tasa de descuento temporal alta o con un área bajo la curva cercana a cero y una tasa de descuento probabilístico baja o con un área bajo la curva cercana a uno, reflejarían a un sujeto impaciente y propenso a las situaciones de riesgo; un perfil similar al que se describe en los manuales de diagnóstico de abuso de sustancias. En la figura 3 se presentan las áreas bajo la curva para el descuento temporal y probabilístico de dinero (triángulos blancos) y para el alcohol (círculos negros) de los participantes en los que se observó descuento sistemático para la misma recompensa, en ambos tipos de descuento. En este caso, 20 participantes mostraron descuento temporal y probabilístico sistemático

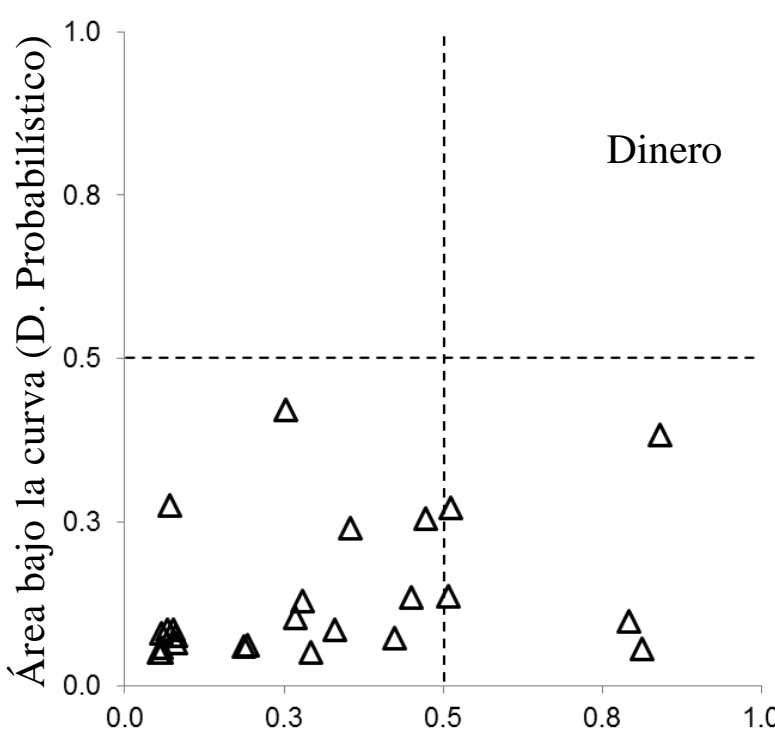

Área bajo la curva (D. Temporal) cuando la recompensa era dinero y 10 cuando la recompensa era alcohol. Las líneas punteadas ilustran la ubicación de los cuadrantes en cada panel, pero no representan ningún valor cuantitativo.

Para cada panel de la figura, en los cuadrantes superiores e inferiores se encuentran los participantes con alta o baja propensión al riesgo, respectivamente. En los cuadrantes izquierdos se ubican los participantes impacientes y en los cuadrantes derechos, aquellos inclinados a comportarse pacientemente. En ambos tipos de recompensa, se observa que la mayoría de los participantes se agrupa en el cuadrante inferior izquierdo, con áreas bajo la curva cercanas a cero para ambos tipos de descuento. Es decir, los participantes del presente estudio presentarían conductas impacientes, pero poco riesgosas, sin importar el tipo de recompensa que obtendrían.

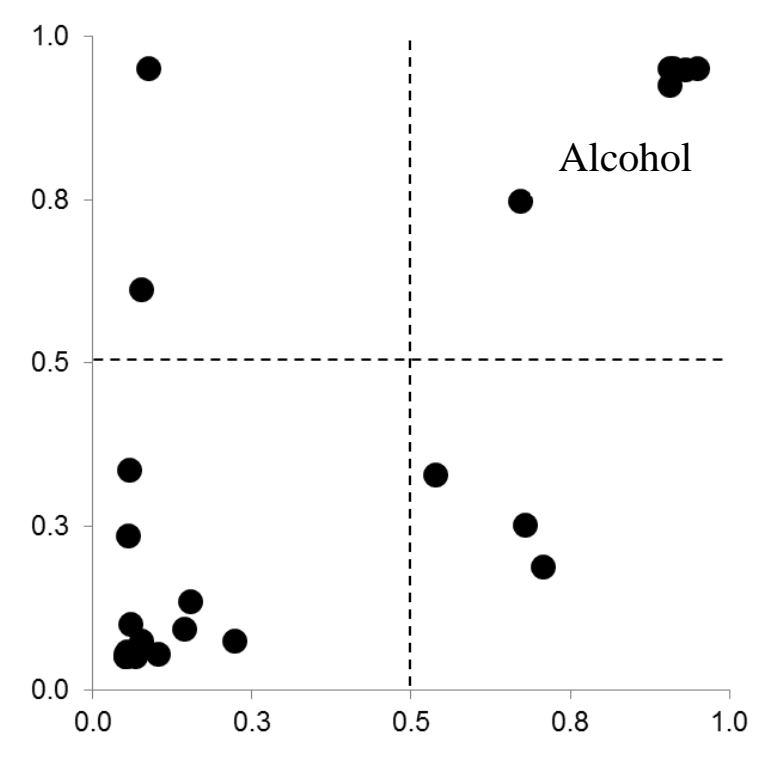

Figura 3. Áreas bajo la curva para el descuento probabilístico en función del descuento temporal de dinero (triángulos) y alcohol (círculos); se presentan los datos de los participantes con descuento sistemático para la misma recompensa. Las líneas punteadas ilustran los cuadrantes, pero no representan valores cuantitativos. 


\section{Discusión}

En este trabajo se comparó la tasa de descuento temporal y probabilístico de recompensas económicas y consumibles, ambas hipotéticas, en usuarios con problemas de abuso de alcohol, quienes respondieron a la tarea de descuento al inicio de un programa para dejar el consumo.

Se encontró que la función hiperbólica ajustó adecuadamente los datos para cada una de las recompensas cuando se tomaron las medianas de los puntos de indiferencia de los participantes. $\mathrm{La}$ demostración de la utilidad de la función hiperbólica para describir las curvas de descuento apoya los resultados de investigaciones previas en las cuales se ha probado su generalidad (Green y Myerson, 2004, 2010). En el caso del descuento temporal, cuando se compararon las tasas de descuento obtenidas de las medianas de los 25 participantes, se encontró una curva ligeramente más acelerada para el alcohol en comparación con el dinero. A primera vista este resultado es consistente con los hallazgos de Petry (2001), quien reportó que personas sin historial de consumo de sustancias y personas con problemas de abuso de alcohol descuentan con mayor facilidad una recompensa consumible que una recompensa económica. Por su parte, Odum y Rainaud (2003) y Estle et al. (2007) reportaron que personas sin problemas de consumo de sustancias descontaron más una recompensa consumible en comparación con una recompensa no consumible. Por lo tanto, pareciera que la magnitud con que se descuenta una recompensa en función de su demora de entrega depende de la naturaleza misma de la recompensa y no de una característica del participante, en este caso, el consumo problemático de sustancias adictivas. En el caso del descuento probabilístico se encontró que las tasas de descuento hiperbólico con los 25 participantes para el dinero y para el alcohol fueron prácticamente iguales. Este resultado es similar al reportado por Estle et al. (2007) quienes encontraron que las tasas de descuento probabilístico de personas sin problemas de consumo de sustancias son similares, independientemente de la naturaleza de la recompensa.

Tanto en el descuento temporal como en el descuento probabilístico, al calcular las tasas de descuento individuales para comparar estadísticamente las tasas de descuento de dinero y alcohol se encontraron varios problemas. El primero de ellos fue que el modelo hiperbólico no pudo explicar los datos individuales de todos los participantes en los dos tipos de descuento y los dos tipos de recompensa. Para resolverlo, se utilizó el análisis de área bajo la curva propuesto por Myerson et al. (2001), un análisis teóricamente neutral, en el cual la forma de la curva de indiferencia no debe ajustarse a un modelo matemático (e.g., Ecuación 1 o Ecuación 2); la estimación del descuento de una recompensa se hace a partir del espacio existente entre el eje de las abscisas y la curva misma. Con este análisis también se busca eliminar el sesgo en la distribución de las tasas de descuento para comparar entre grupos de datos. Otros autores han buscado estrategias alternas, como Petry (2001) quien reportó que para realizar los análisis estadísticos normalizó con logaritmos la distribución de las tasas de descuento.

A pesar de que con el área bajo la curva pueden analizarse los puntos de indiferencia de todos los participantes, se ha reportado que en algunos casos los participantes no descuentan sistemáticamente el valor de una recompensa. En el presente trabajo se identificaron casos en los cuales los participantes no descontaron el 
valor de la recompensa en función de su demora o de su probabilidad de entrega. Johnson y Bickel (2008) describieron el descuento no sistemático como aquellas situaciones en las cuales los participantes pueden no estar atentos a la tarea, pueden no haber entendido cómo responder o simplemente respondieron de manera desinteresada. A pesar de que en las instrucciones de la tarea se incluyeron ensayos de prueba para asegurar que los participantes entendieron de qué se trataba, posiblemente incluir bebidas alcohólicas como recompensa para participantes que trataban de dejar de beber influyó en las respuestas durante la tarea. En una futura investigación debe considerarse la posibilidad de incluir recompensas consumibles no alcohólicas, para controlar efectos de deseabilidad experimental o desinterés por participar en la tarea.

Un resultado obtenido en el presente trabajo distinto al observado en la literatura de descuento temporal es que no se encontraron diferencias significativas entre las áreas bajo la curva de alcohol y dinero (Estle et al., 2007; Odum y Rainaud, 2003). A pesar de ello, la tendencia de los datos aquí presentados es la misma que se ha reportado en la literatura; esto es, que los participantes descuentan más el valor del alcohol en comparación con el descuento al valor del dinero. La falta de diferencias estadísticas puede explicarse por la variabilidad que se observó en los datos y por el reducido número de participantes en los que se encontró descuento sistemático (13 de 25). En el caso del descuento probabilístico se encontró que no hay diferencia entre las áreas bajo la curva del alcohol y del dinero. Con este resultado se replicaron los hallazgos previos (Estle et al., 2007) y se extienden a participantes con consumo problemático de alcohol. Una futura investigación deberá corroborar estos hallazgos e incluir comparaciones con poblaciones sin historial de consumo de sustancias y con participantes con historial de consumo en abstinencia (Petry, 2001).

En términos de la relación entre el descuento temporal y el descuento probabilístico, algunos autores han propuesto que tanto el descuento probabilístico como el descuento temporal registran aspectos similares de la conducta impulsiva (e.g., Jones y Rachlin, 2009). Para otros autores, el descuento temporal y el probabilístico están relacionados, pero no necesariamente el proceso involucrado es el mismo (e.g., Estle et al., 2007; Green y Myerson, 2010). Esto es, si el descuento temporal y el descuento probabilístico son el resultado del mismo proceso, las diferencias en las tasas de descuento de dinero y alcohol deberían mantenerse. En el presente trabajo, se observaron diferencias sin significancia estadística entre las tasas de descuento temporal y tasas de descuento probabilístico prácticamente iguales. A partir de estos resultados y los reportes en la literatura, aparentemente cada tipo de descuento registra un patrón de conducta específico de una conducta general más grande que se conoce como impulsividad. En el caso del descuento temporal es posible registrar conductas que van desde la impaciencia hasta la paciencia, por ejemplo, si se elige la recompensa inmediata o la recompensa demorada. En el caso del descuento probabilístico se registran conductas relacionadas con la propensión o la aversión al riesgo, que son respuestas ante situaciones que involucran o no incertidumbre en la obtención de una recompensa. De este modo, es difícil integrar ambos patrones de conducta en una definición de impulsividad que incluya la demora y la probabilidad de entrega de la recompensa (Green y Myerson, 2013). 
Es necesario explorar con mayor detalle la relación entre el descuento temporal y el descuento probabilístico con el consumo de sustancias; posiblemente comprender la forma en que los usuarios de sustancias descuentan el valor de una recompensa puede ser útil para entender los procesos y los mecanismos involucrados en la adicción a las sustancias psicoactivas y las posibles consecuencias o resultados de un tratamiento. Por ejemplo, con las tasas de descuento temporal probablemente se pueda predecir el tiempo que el usuario está dispuesto a esperar para obtener resultados positivos en un tratamiento. Por otro lado, las tasas de descuento probabilístico pueden servir para predecir qué tan probable es que el usuario prefiera involucrarse en una conducta de riesgo como podría ser continuar con el consumo de sustancias. Si al aplicar tareas de descuento a lo largo del tratamiento se observan cambios ordenados en las tasas de descuento conforme cambian los patrones de consumo de sustancias, posiblemente pueda entenderse mejor la conducta impulsiva de los usuarios de drogas para, eventualmente, predecir la efectividad de un tratamiento determinado.

\section{Referencias}

American Psychiatric Association, APA. (2000). Manual diagnóstico y estadístico de los trastornos mentales, cuarta edición revisada. Washington, DC: Autor.

Bickel, W. K., Odum, A. L., \& Madden, G. J. (1999). Impulsivity and cigarette smoking: Delay discounting in current, never, and ex-smokers. Psychopharmacology, 146, 447-454. http://dx.doi.org/10.1007/PL00005490

Estle, S. J., Green, L., Myerson, J., \& Holt, D. D. (2007). Discounting of monetary and directly consumable rewards. Psychological Science, 18, 58-63. http://dx.doi.org/10.1111/j.1467-9280.2007.01849.x

Franck, J. \& Jayaram-Lindström, N. (2013). Pharmacotherapy for alcohol dependence: Status of current treatments. Current Opinion in Neurobiology, 23, 692-699. http://dx.doi.org/10.1016/j.conb.2013.05.005

Green, L. \& Myerson, J. (2004). A discounting framework for choice with delayed and probabilistic rewards. Psychological Bulletin, 130, 769-792. http://dx.doi.org/10.1037/0033-2909.130.5.769

Green, L. \& Myerson, J. (2010). Experimental and correlational analyses of delay and probability discounting. In G. J. Madden y W. K. Bickel (Eds.), Impulsivity: The behavioral and Neurological Science of Discounting (pp. 67-92). Washington, DC: American Psychological Association. http://dx.doi.org/10.1037/12069-003

Green, L. \& Myerson, J. (2013). How many impulsivities? A discounting perspective. Journal of the Experimental Analysis of Behavior, 99, 3-13. http://dx.doi.org/10.1002/jeab.1

Instituto Nacional de Psiquiatría Ramón de la Fuente Muñiz, Instituto Nacional de Salud Pública, Secretaría de Salud. (2012). Encuesta nacional de adicciones 2011. Reporte de alcohol. Recuperado de http://www.conadic.salud.gob.mx/ 
Johnson, M. W. \& Bickel, W. K. (2008). An algorithm for identifying nonsystematic delaydiscounting data. Experimental and Clinical Psychopharmacology, 16, 264-274. http://dx.doi.org/10.1037/1064-1297.16.3.264

Jones, B. A. \& Rachlin, H. (2009). Delay, probability, and social discounting in a public goods game. Journal of Experimental Analysis of Behavior, 91, 61-73. http://dx.doi.org/10.1901/jeab.2009.91-61

Logue, A. W. (1988). Research on self-control: An integrating framework. Behavioral and Brain Sciences, 11, 665-679. http://dx.doi.org/10.1017/S0140525X00053978

Myerson, J., Green, L., \& Warusawitharana, M. (2001). Area under the curve as a measure of discounting. Journal of Experimental Analysis of Behavior, 76, 235-243. http://dx.doi.org/10.1901/jeab.2001.76-235

Odum, A. L. \& Rainaud, C. P. (2003). Discounting of delayed hypothetical money, alcohol and food. Behavioral Processes, 64, 305-313. http://dx.doi.org/10.1016/S0376-6357(03)00145-1

Organización Mundial de la Salud, OMS. (2001). Cuestionario de identificación de los trastornos debidos al consumo de alcohol. Ginebra: Autor.

Organización Mundial de la Salud, OMS. (2014). Global status report on alcohol and health. Ginebra: Autor.

Petry, N. M. (2001). Delay discounting of money and alcohol in actively using alcoholics, currently abstinent alcoholics, and controls. Psychopharmacology, 154, 243-250. http://dx.doi.org/10.1007/s002130000638

Rachlin, H., Raineri, A., \& Cross, D. (1991). Subjective probability and delay. Journal of the Experimental Analysis of Behavior, 55, 233-244.

http://dx.doi.org/10.1901/jeab.1991.55-233

Richards, J. B., Zhang, L., Mitchell, S. H., \& de Wit, H. (1999). Delay or probability discounting in a model of impulsive behavior: Effect of alcohol. Journal of Experimental Analysis of Behavior, 71, 121-143.

http://dx.doi.org/10.1901/jeab.1999.71-121

Yi, R., Mitchell, S. H. \& Bickel, W. K. (2010). Delay discounting and substance abusedependence. In G. J. Madden y W. K. Bickel (Eds.), Impulsivity: The behavioral and neurological science of discounting (pp. 191-211). Washington, DC: American Psychological Association.

http://dx.doi.org/10.1037/12069-007 